\title{
Development and validation of an rDNA operon based primer walking strategy applicable to de novo bacterial genome finishing
}

\author{
Alexander W. Eastman ${ }^{1,2}$ and Ze-Chun Yuan ${ }^{1,2 *}$ \\ ${ }^{\prime}$ Southern Crop Protection and Food Research Centre, Agriculture and Agri-Food Canada, Government of Canada, London, ON, Canada \\ 2 Department of Microbiology and Immunology, Schulich School of Medicine and Dentistry, University of Western Ontario, London, ON, Canada
}

Edited by:

Frank T. Robb, University of

Maryland, USA

Reviewed by:

Jonathan H. Badger, J. Craig Venter

Institute, USA

Viktoria Shcherbakova, Russian

Academy of Sciences, Russia

\section{*Correspondence:}

Ze-Chun Yuan, Southern Crop

Protection and Food Research

Centre, Agriculture and Agri-Food

Canada, 1391 Sandford Street,

London, ON N5V 4T3, Canada

e-mail:zyuan27@uwo.ca
Advances in sequencing technology have drastically increased the depth and feasibility of bacterial genome sequencing. However, little information is available that details the specific techniques and procedures employed during genome sequencing despite the large numbers of published genomes. Shotgun approaches employed by second-generation sequencing platforms has necessitated the development of robust bioinformatics tools for in silico assembly, and complete assembly is limited by the presence of repetitive DNA sequences and multi-copy operons. Typically, re-sequencing with multiple platforms and laborious, targeted Sanger sequencing are employed to finish a draft bacterial genome. Here we describe a novel strategy based on the identification and targeted sequencing of repetitive rDNA operons to expedite bacterial genome assembly and finishing. Our strategy was validated by finishing the genome of Paenibacillus polymyxa strain CR1, a bacterium with potential in sustainable agriculture and bio-based processes. An analysis of the 38 contigs contained in the P. polymyxa strain CR1 draft genome revealed 12 repetitive rDNA operons with varied intragenic and flanking regions of variable length, unanimously located at contig boundaries and within contig gaps. These highly similar but not identical rDNA operons were experimentally verified and sequenced simultaneously with multiple, specially designed primer sets. This approach also identified and corrected significant sequence rearrangement generated during the initial in silico assembly of sequencing reads. Our approach reduces the required effort associated with blind primer walking for contig assembly, increasing both the speed and feasibility of genome finishing. Our study further reinforces the notion that repetitive DNA elements are major limiting factors for genome finishing. Moreover, we provided a step-by-step workflow for genome finishing, which may guide future bacterial genome finishing projects.

Keywords: genome finishing, ribosomal DNA, contigs assembly, bacterial genomics, second-generation sequencing (SGS)

\section{INTRODUCTION}

The advent of high-throughput second-generation sequencing platforms has allowed for a monumental leap in the affordability and feasibility of genomic studies (Bentley, 2010; Delseny et al., 2010). Applications of these second-generation sequencing technologies have been especially helpful and disruptive during the characterization of microorganisms, where whole genome sequencing has become a routine step in bacterial strain characterizations (Medini et al., 2008; Maclean et al., 2009).

Four second-generation sequencing platforms are commonly employed for de novo sequencing of bacterial genomes; Illumina, 454 Roche, SOLiD and Ion Torrent. With the exception of SOLiD, second-generation sequencing technologies determine the sequence of short, fragmented DNA molecules through detection of nucleotides incorporated during synthesis of a complementary strand. Each technology varies in the specifics of the sequencing chemistry, read-length, library preparation and output accuracy, and the advantages and disadvantages of each specific technology have been reviewed previously (Miller et al., 2008; Glenn, 2011; Quail et al., 2012; McGinn and Gut, 2013; Morey et al., 2013).

In second-generation sequencing platforms, sequencing reads are assembled by computational assignment of nucleotide identities. Where applicable, software trims the known adaptor sequences from the DNA sequences, and removes bases and reads that do not meet quality thresholds and in silico assembly algorithms are used to assemble the remaining reads into contiguous sequences (contigs) (Pop et al., 2004; Horner et al., 2009; Gritsenko et al., 2012). Detailed methods using the power of second-generation sequencing platforms for various experimental aims are available; however there are few examples in the literature describing the finishing procedures for bacterial 
genomes. De novo assembly of a bacterial genome results in multiple contigs ranging in length from a few hundred to thousands of base pairs, commonly referred to as a draft genome (Tsai et al., 2010; Wetzel et al., 2011). Taken alone, contigs contained within a draft genome offer no spatial orientation relative to each other and are numbered in descending order by size. In addition, draft genomes are error-prone, containing frame shifts, missing genes/sequence, rearrangements, ambiguous bases, and sequencing artifacts and do not always accurately reflect genome structure (Poptsova and Gogarten, 2010; Ricker et al., 2012). The increased accuracy and fidelity of completely sequenced and finished genomes are preferential to draft genomes for later use in systems biology and the various "omics" fields.

Paenibacillus polymyxa, the type species of the Paenibacillus genus, have been isolated from diverse environments including marine sediments, hot-springs and soils world-wide and are commonly found in association with plant hosts. $P$. polymyxa strains are best known for promoting plant fitness through pathogen antagonism, nutrient cycling, and priming of plant defenses (Lal and Tabacchioni, 2009). Recently, interest for potential applications in agriculture and renewable bio-fuel production has driven the resurgence in studies of $P$. polymyxa biology (Yu et al., 2011; Eastman et al., 2014b). Besides our sequencing of $P$. polymyxa strain CR1 (here forth, CR1), complete genomes of four other P. polymyxa strains (E681, SQR-21, M1, and SC2) are publically available among numerous draft sequences (Kim et al., 2010; Ma et al., 2011; Niu et al., 2011; Eastman et al., 2014a; Li et al., 2014).

Here we detail the sequencing, assembly, and finishing of the CR1 genome using a modified primer walking strategy developed to minimize efforts for gap closure. Our approach simplifies the sequencing procedure for repetitive rDNA operons, thereby speeding up genome sequencing, assembly, and finishing. Furthermore, we illustrate the most common approach to bacterial genome sequencing and offer an overview of the challenges of bacterial genome finishing.

\section{METHODS AND RESULTS SECOND-GENERATION SEQUENCING}

Whole genome sequencing using second-generation sequencing technologies is a powerful tool for microbiologists looking to identify the potential genetic basis of observed phenotypes. Prior to proceeding with completing a draft bacterial genome, the quality of the sequencing run and draft assembly must be taken into careful consideration. Adequate genome coverage of the sequencing read libraries are critical for assembling a genome with high fidelity. Typically, 40 50× coverage is needed for genome sequencing and higher coverage $(>50 \times)$ does not necessarily increase assembly strength. If the sequencing depth is low it is advisable to re-sequence the genome since the power of second-generation sequencing highly relies on high coverage to overpower the limitations of short read lengths and comparatively low individual base accuracy. Currently the $\mathrm{Q}_{30}$ score is the de facto standard for measuring the accuracy of secondgeneration reads, a $Q_{30}$ score represents a $1 / 1000$ chance of an incorrect base identification (Ewing and Green, 1998; Ewing et al., 1998). It is important to note that $Q_{30}$ is presented as a percentage of total bases in the sequencing run that reach the threshold and typically, $\mathrm{Q}_{30}$ scores for a high-quality run are $>80 \%$. In the case of the CR1 genome, sequencing was performed using the Illumina MiSeq platform. A short-insert read library was generated with a target insert size of $400 \mathrm{bp}$ using the NexteraXT DNA sample preparation kit and a mate-pair library was generated using the Nextera mate-pair sample preparation kit. MiSeq $2 \times 150$ bp sequencing yielded a $140 \times$ genome coverage short-insert read library (2.9 million reads) and a $107 \times$ genome coverage mate pair read library (2.2 million reads). Adaptor sequences were removed, mate-pair reads with insert sizes $>400$ base pairs were filtered and the two libraries were merged generating a $40 \times$ genome coverage merged library with a mean mate-pair insert size of $1.25 \mathrm{~kb}$ and a $\mathrm{Q}_{30}$ score of $86 \%$ (Figure 1).

\section{GENOME DRAFT ASSEMBLY OF P. POLYMYXA STRAIN CR1}

Concomitant with the advances of second-generation sequencing, developments of in silico assembly programs has yielded numerous bioinformatics tools designed to minimize and compensate for sequencing errors inherent to each respective assembly platform. Each algorithm varies the length of matching sequence required before two reads are considered overlapping (called the k-mer length), which has a dramatic effect on the generated contig assembly. A short k-mer length results in a more contiguous assembly at the cost of accuracy and fidelity, while a long k-mer length results in a highly fragmented but more accurate draft assembly (Gibbons et al., 2009). Recent tools have been developed to integrate the outputs from multiple assembly programs each with different k-mer lengths into a single set of contigs with higher strength and accuracy. The advantage of integrating the contigs generated by multiple programmes stems from the ability to combine the highly accurate, long k-mer assemblies with the less accurate but more contiguous short k-mer assemblies. In the case of CR1 genome sequencing, three separate contig assembly programs, ABySS with a k-mer length of $67 \mathrm{bp}$ (Birol et al., 2009), Velvet with a k-mer length of 31 bp (Zerbino and Birney, 2008), and SOAPdenovo with a k-mer length of 55 bp (Luo et al., 2012), were run independently, generating three separate contig assemblies that are then integrated into a final draft assembly by CISA (Lin and Liao, 2013). The draft assembly of the CR1 genome contained 38 contigs with an $\mathrm{N}_{50}$ value of $1.5 \mathrm{Mb}\left(N_{50}=\right.$ the size of contig where $50 \%$ of the total bases are accounted for when combined with all longer contigs), representing an excellent draft genome for finishing. It may be wise to re-sequence the genome prior to finishing if the sequencing read library has poor genome coverage, a low $\mathrm{Q}_{30}$-score or the draft genome is highly fragmented ( $>100$ contigs), especially in cases where no closely related strains or species are available as reference genomes for scaffold assembly.

Regardless of which technology is used to generate the draft genomic data, the procedure for genome assembly and finishing are more or less analogous (Nagarajan et al., 2010). Unfortunately, no automated/high-throughput and universally applicable method has been developed for finishing of de novo bacterial genomes and Sanger sequencing remains the most commonly used approach (Schuster, 2008; Hurt et al., 2012). Finishing of bacterial genomes typically involves five major steps 


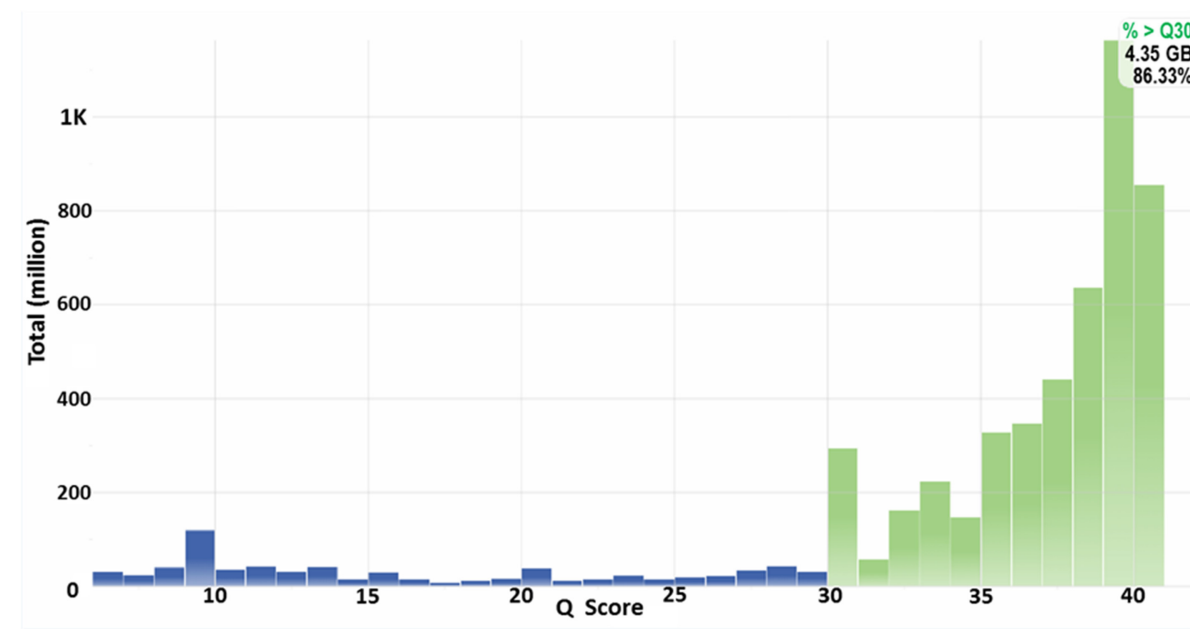

FIGURE 1 | $\mathbf{Q}_{\mathbf{3 0}} \mathbf{g r a p h}$ of Illumina reads. Phred-like quality scores (Q-scores) are used to measure the accuracy of nucleotide identity data from a sequencing run. A higher quality score indicates a lower probability that an individual base is called incorrectly, where $\mathrm{Q}=-10_{\log } 10(\mathrm{e}) \cdot \mathrm{Q}_{20}$ indicates the probability of an incorrect base call is 1 in 100; $\mathrm{Q}_{30}, 1$ in 1000; $\mathrm{Q}_{40}, 1$ in 10,000 The green proportion of the graph represents bases with a high quality score (defined as $\mathrm{Q}_{30}$ ) identified during the run. The whole genome de novo sequencing of Paenibacillus polymyxa strain $\mathrm{CR} 1$ had $86.33 \%=\mathrm{Q}_{30}$ bases. after the assembly of a draft genome (Figure 2); locating and sequencing of ambiguous bases, scaffold assembly of contigs, investigation of contig rearrangements, sequencing of contig gaps, and genome annotation.

\section{REDUCTION OF rRNA CONTIGS BY IN SILICO ALIGNMENT}

Repetitive genetic elements are known to be causative of misassembled contigs during de novo genome assembly (Ricker et al., 2012). Bacterial genomes encode multiple, highly-similar operons encoding $16 \mathrm{~S}, 23 \mathrm{~S}$, and $5 \mathrm{~S}$ ribosomal subunits, commonly referred to as rDNA operons (Rainey et al., 1996). Manual inspection of contig ends determined that various short contigs $(<2 \mathrm{~kb})$ corresponded to isoforms of rRNA genes. Contigs comprised of multi-copy repetitive elements such as rDNA have the potential to be assembled from sequencing reads corresponding to distant genomic loci and are thus prone to being chimeric. We employed Sanger sequencing with universal $16 \mathrm{~S}$ and $23 \mathrm{~S}$ rDNA primers (Table 1) to generate consensus sequences for both the $16 \mathrm{~S}$ and 23S genes (Anzai et al., 2000; Hunt et al., 2006). Alignment of the consensus sequences against the 38 contigs from the CR1 draft genome allowed for masking of 10 short contigs ( $<2 \mathrm{~kb})$ that corresponded to various isoforms of rRNA genes. Thus, assembly and masking of the short contigs containing highly similar rDNA into consensus $16 \mathrm{~S}$ and $23 \mathrm{~S}$ genes reduced the set of contigs from 38 to 28 . Within this set of 28 contigs, 24 of the remaining ends (of a possible 56 ends) contained some fragment of 5S, 16S, or $23 \mathrm{~S}$ rRNA genes.

\section{SCAFFOLDING OF DRAFT GENOME}

The explosion of second-generation sequencing projects has dramatically increased the number of published genomes from all walks of life, especially prokaryotes. Accordingly the availability of a closely related sequence facilitates scaffolding of newly obtained draft genomes against a previously completed, closely related genome, identifying putative gaps and variability in the sequence for experimental investigation. The multiple genome alignment software Mauve iteratively compares segments of DNA to user-provided genomes by characterizing regions of high similarity as local collinear blocks, which can then be used to reorder the contigs to generate the best assembly compared to any user-provided reference genome (commonly referred to as scaffolding) (Darling et al., 2010, 2011). In the case of the CR1 genome assembly, the draft genome with 28 remaining contigs was aligned using progressiveMauve against the completely sequenced genome of $P$. polymyxa strain E681 which showed the highest nucleotide-level similarity to CR1 (Kim et al., 2010). With the reference genome as scaffold, we were able to draft the final CR1 genome assembly and identify putative contig gaps for further sequencing and closure. For de novo sequencing projects where the bacterium is the first member of its species to be sequenced, the next closest related species may be used for scaffold assembly. For example, alignments of the CR1 draft genome against the complete Paenibacillus terrae strain HPL-003 genome (Shin et al., 2012) also resulted in a contig assembly that approximated the alignment against $P$. polymyxa strain E681 (Figure 3).

\section{TARGETED SEQUENCING OF AMBIGUOUS BASES}

Reduction of a highly fragmented draft genome comprised of numerous contigs to a single contiguous sequence is the most noticeable feature of finished genomes (Mardis et al., 2002). However, genomes sequenced using paired-end runs and assembled using Velvet or ABySS occasionally include series of ambiguous bases within the assembled contigs, represented by $\mathrm{N}$ nucleotides in FASTA formatted data (Mardis et al., 2002; Zerbino and Birney, 2008; Birol et al., 2009). The lengths of ambiguous base stretches can range from three to hundreds of bases in length and are determined computationally during read assembly. These ambiguous base stretches can result from mate-pair reads without sufficient internal coverage, nucleotide polymorphisms and 


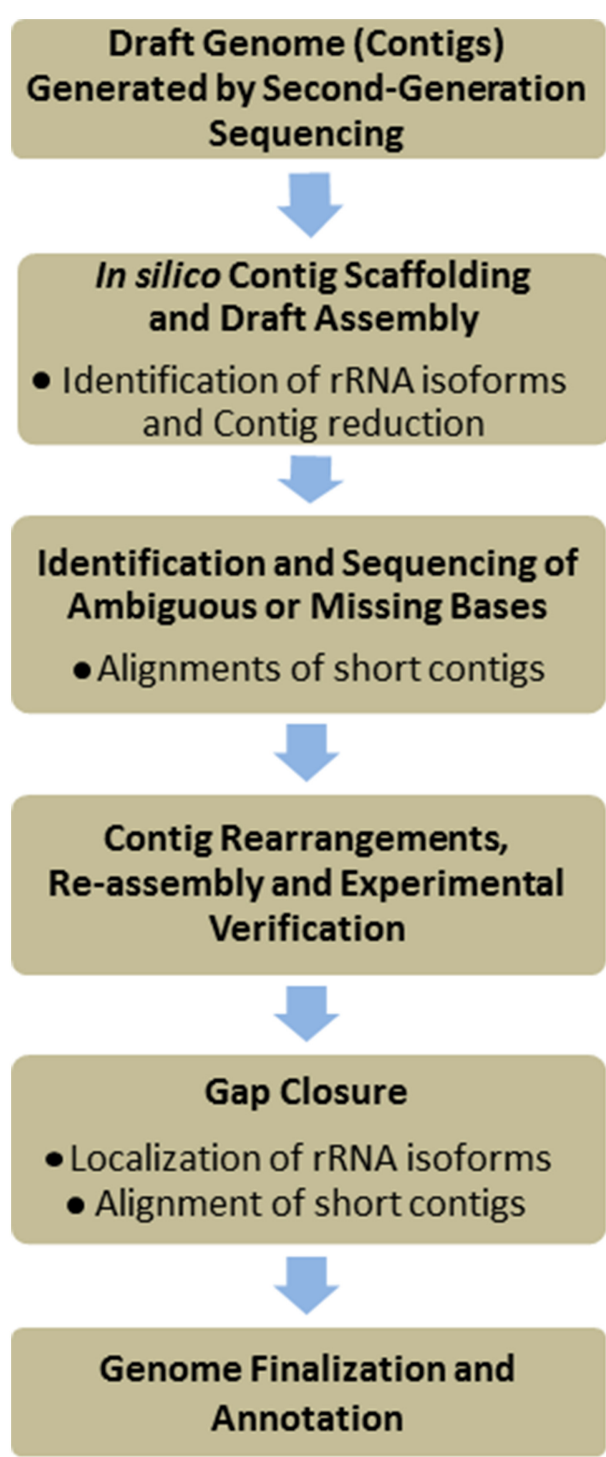

FIGURE 2 | Flow chart for de novo bacterial genome finishing. The assembly process is initially simplified by removal of contigs comprised entirely of rRNA genes. The draft genome is then scaffolded against other closely related strains and species to identify potential gaps, rearrangements, and missing sequences. Ambiguities in structure and nucleotide identity are targeted for Sanger re-sequencing. Furthermore, large scale rearrangements are carefully investigated through a combination of PCR and sequencing to ensure mistakes are not incorporated during draft genome assembly. Finally gaps between contigs are sequenced and the genome is assembled and annotated.

individual bases within reads that do not meet quality thresholds. Nevertheless, ambiguous bases should be targeted for Sanger sequencing to fill in the missing bases and can be treated as short, pre-defined contig gaps during sequencing. The draft genome (28 contigs) of CR1 contained a total of 198 ambiguous base stretches, between 9 and 769 bp in length, distributed irregularly throughout the genome (Figure 4). The generated draft assembly and locations of ambiguous bases were annotated in Artemis (Rutherford et al., 2000) and visualized using DNAplotter (Carver et al., 2009). To identify ambiguous bases, 198 sets of primers flanking the $5^{\prime}$ and $3^{\prime}$ ends of each ambiguous base stretch were designed. Each primer set was used to amplify the ambiguous base stretch by PCR using the following cycle; $95^{\circ} \mathrm{C}$ for $1 \mathrm{~min}$ followed by 35 cycles of $95^{\circ} \mathrm{C}$ for $30 \mathrm{~s}$, varied annealing temperature dependent on $\mathrm{T}_{\mathrm{m}}$ of the primer pair for $45 \mathrm{~s}, 72^{\circ} \mathrm{C}$ for $60 \mathrm{~s}$ followed by a final extension at $72^{\circ} \mathrm{C}$ for $5 \mathrm{~min}$. To ensure each PCR product corresponded to the intended ambiguous bases location, 198 PCR products were individually sequenced bi-directionally and aligned against the CR1 draft genome (28 remaining contigs) to verify the location of each ambiguous base stretch and fill in the missing sequence. Interestingly, sequences of $10 \mathrm{ambigu-}$ ous base stretches matched with the sequence of 10 small contigs $(<1 \mathrm{~kb}$ in size), indicating these 10 small contigs actually fit within these 10 ambiguous base stretches. Thus, sequencing of ambiguous base stretches reduced the set of contigs from 28 to 18 , where 10 small contigs merged into 10 ambiguous base stretches.

\section{NOVEL GAP CLOSURE APPROACH}

For gap closure of the CR1 draft genome, gaps (average length of $7.5 \mathrm{~kb}$ ) between the 18 remaining contigs were identified through Mauve alignments against the complete genomes of $P$. polymyxa strain E681 and P. terrae strain HPL-003 (Figures 3, 4). Contig gaps were amplified from CR1 genomic DNA using oligonucleotides designed to hybridize between 250 and $350 \mathrm{bp}$ from the $5^{\prime}$ and $3^{\prime}$ ends of each contig. PCR conditions for amplification of contig gaps were as follows; $95^{\circ} \mathrm{C}$ for $1 \mathrm{~min}$ followed by 40 cycles of $95^{\circ} \mathrm{C}$ for $30 \mathrm{~s}$, varied annealing temperature dependent on $\mathrm{T}_{\mathrm{m}}$ of the primer pair for $45 \mathrm{~s}, 72^{\circ} \mathrm{C}$ for $300 \mathrm{~s}$ followed with a final extension at $72^{\circ} \mathrm{C}$ for $20 \mathrm{~min}$. All PCR amplifications of contig gaps were performed using Phusion ${ }^{\circledR}$ Taq polymerase from NEB and products were excised and gel-purified. Absence of contaminating genomic DNA in gel-purified contig gap product was confirmed using primer sets corresponding to distant genomic DNA locations. During our masking of contigs comprised entirely of rDNA we noticed the prevalence of fragments of rDNA at contig boundaries. Since rRNA operons are multicopy in any given bacterial genome, universal primers targeting $16 \mathrm{~S}$ and $23 \mathrm{~S}$ rRNA genes (Table 1) were used for PCR to determine if a candidate amplified gap (gel-purified PCR products as template) contained rDNA (Figures 5, 6). Through this method, we identified 12 out of the 18 remaining amplified contig gaps contained an rRNA operon. Their prevalence and localization to contig boundaries is likely a consequence of short read sequencing technology that cannot sequence across the entirety of an rRNA operon. For instance, the gaps between contigs 10 and 3 (10/3), contigs 5 and $6(5 / 6)$, and contigs 8 and 4 (8/4) contain both $16 \mathrm{~S}$ and $23 \mathrm{~S}$ rDNA, while gap between contigs 12 and $9(12 / 9)$ does not contain any rDNA (Figure 6). These results further confirmed our earlier assumption that the repetitive nature of rRNA operons was preventing a more complete in silico assembly.

Traditional primer walking involves Sanger sequencing using a primer complementary to the end of known sequence, resulting in approximately $750 \mathrm{bp}$ to $1 \mathrm{~kb}$ of readable sequence per sequencing run. New primers are then designed to hybridize to 
Table 1 | Universal $16 S$ and $23 S$ primers for rapid rRNA operon gap sequencing.

\begin{tabular}{|c|c|c|c|c|}
\hline Primer name & Sequence & Gene & Direction & References \\
\hline $1492 R$ & CGTTACCTTGTTACGACTT & $16 \mathrm{~S}$ & Reverse & Anzai et al., 2000 \\
\hline $2241 R$ & ACCGCCCCAGTHAAACT & $23 S$ & Reverse & Hunt et al., 2006 \\
\hline U1 & TGGGATACCACCCTGATCGT & $16 S$ & Reverse & This study \\
\hline U4 & GTCCGCCGCTAGGTTGATTA & $23 S$ & Forward & This study \\
\hline
\end{tabular}

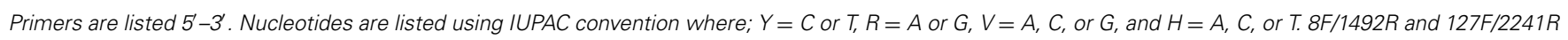
are as previously described by Anzai et al. (2000) and Hunt et al. (2006), respectively. Primers U1, U2, U3, and U4 hybridize to rDNA operons as depicted in Figure 5.

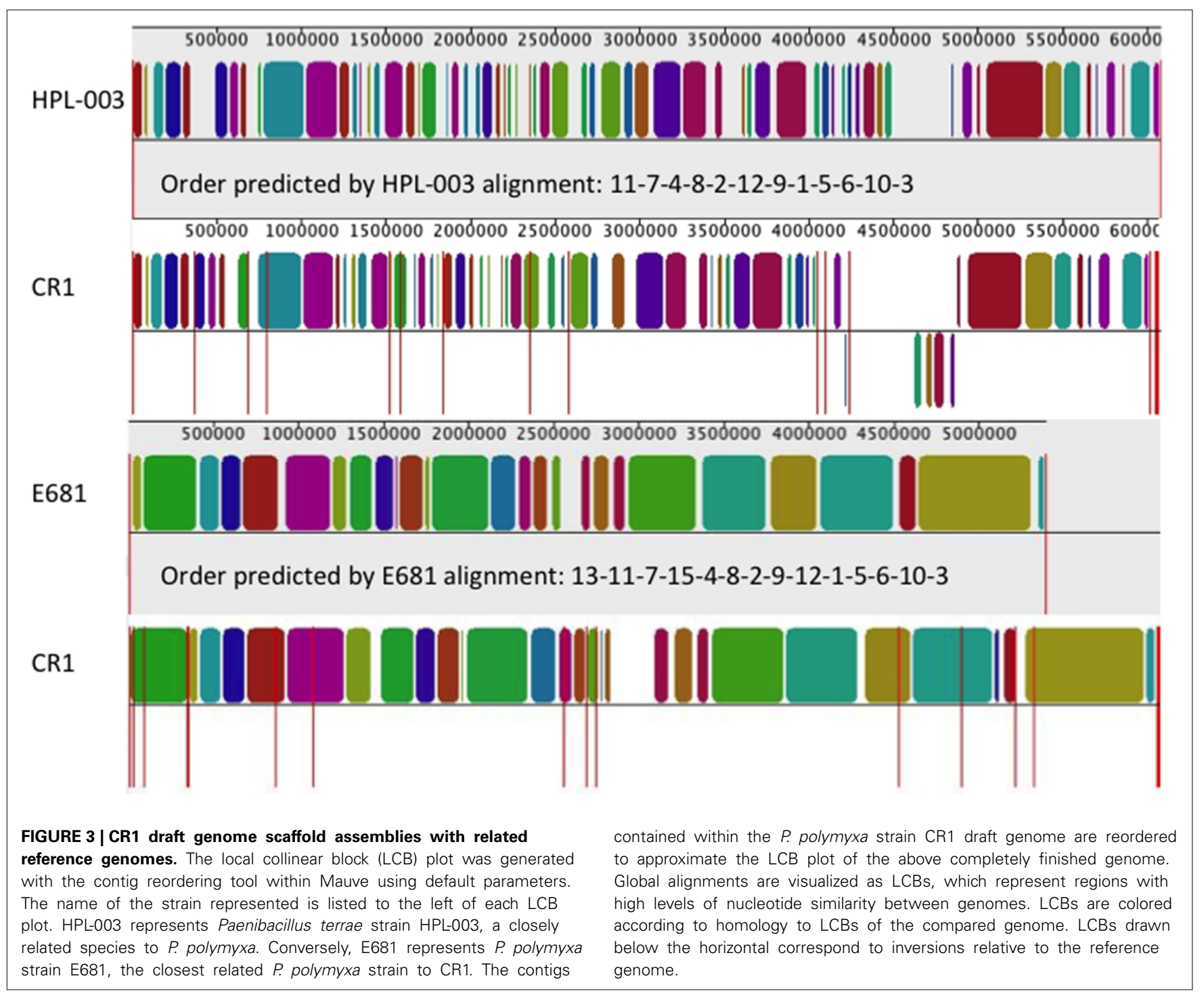

the determined sequence and the process repeats itself until the gap is filled. To speed up gap closure, we designed several sets of rRNA gene specific primers (Primers U1, U2, U3, U4) that hybridize to the $5^{\prime}$ and $3^{\prime}$ end of both the $16 \mathrm{~S}$ and $23 \mathrm{~S}$ genes
(Table 1) so that the entire rDNA operon contained in the contig gaps can be sequenced simultaneously including; $16 \mathrm{~S}$ and $23 \mathrm{~S}$ genes, the 16S/23S intragenic sequence, and gene flanking regions (Figure 5). Therefore, for each rDNA operon, six total sequencing 


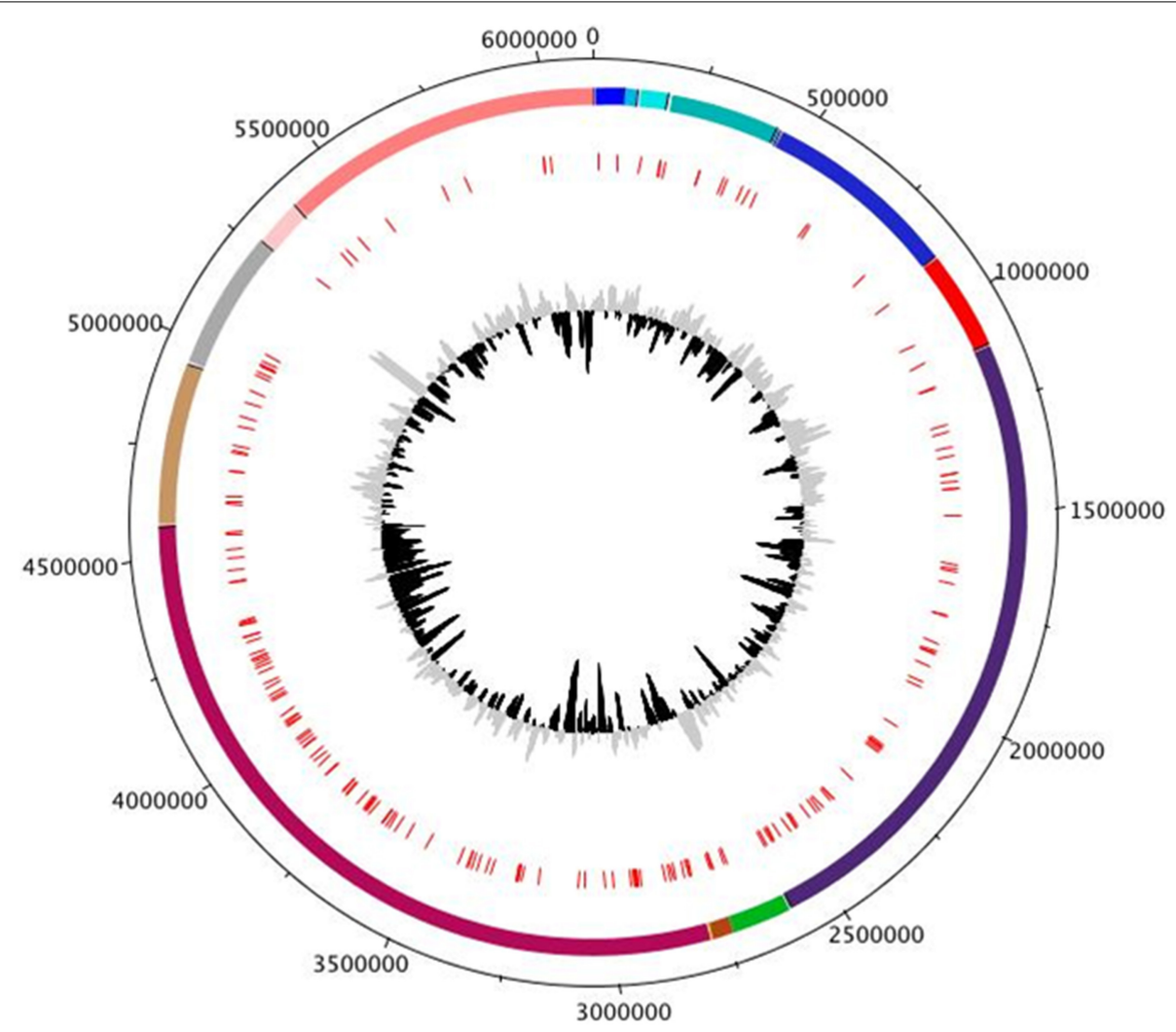

FIGURE 4 | Circular representation of CR1 scaffold assembly showing locations of ambiguous bases. From inside to outside; 1 st ring $-\mathrm{G}+\mathrm{C}$

mol\% content, 2nd ring - locations of ambiguous bases (red), 3rd ring - final assembly of draft contigs where separate contig from the draft assembly are labeled with different colors, 4th ring - genome size in base pairs.

Annotations were performed in Artemis and visualized using DNAplotter. reactions were performed in parallel (equivalent to three stages of primer walking). By taking advantage of the redundant nature of rDNA operons, 10 PCR amplified contig gaps containing rDNA operons were sequenced in parallel by this rDNA based primer walking approach using the designed rRNA gene specific primers (Table 1). In the majority of cases this strategy was sufficient to fill the contig gap in a single step, dramatically increasing the speed of the primer walking procedure. In cases where sequencing results did not reach $16 \mathrm{~S} / 23 \mathrm{~S}$ genes or where the contig gap did not contain $16 \mathrm{~S} / 23 \mathrm{~S}$ genes, traditional primer walking was necessary to fill the remaining gaps.

\section{INVESTIGATION OF REARRANGEMENTS}

One of the most common errors present in draft genomes are rearrangements, a result of incorrect assignment of overlapping reads (Salzberg and Yorke, 2005). Typically, the PCR amplification of ambiguous base stretches during genome finishing is straightforward. For the CR1 genome, difficulties generating PCR product for an ambiguous base stretch located in contig 4 of the draft assembly suggested either an error in the scaffold assembly or a rearrangement in the draft genome. Analyzing low similarity regions in the Mauve alignments of the draft genome also indicated potential rearrangements in the CR1 draft genome assembly (compare local collinear block composition between CR1 and P. polymyxa strain E681, Figure 3). To pinpoint the location of the rearrangements, $10 \mathrm{~kb}$ windows of nucleotide sequence from the CR1 draft genome flanking the putative rearrangements were aligned using BLASTn against completely sequenced genome of P. polymyxa strain E681. Taken together, the results from Mauve alignments and BLASTn alignments suggested a large insertion of the assembled contigs of 11, 13, 7, and 15 immediately adjacent to an ambiguous base stretch in contig 4. Figure 7 offers a schematic representation of the final $P$. polymyxa strain CR1 genome assembly compared to the originally identified in silico assembly and scaffold. To verify the contig rearrangement, we designed the primers $13 \mathrm{~B}$ and $445 \mathrm{~N} 5$ to specifically amplify the joint region of contig 13 and the ambiguous base stretch in contig 4 , as well as specific primers $15 \mathrm{~B}$ and $445 \mathrm{~N} 3$ that flanking the joint region of contig 15 and the ambiguous base stretch in contig 4 (Table 2). As expected, DNA sequences from the generated PCR products confirmed that the rearrangement present in contig 4 of the draft genome was an assembly error. The PCR product from primers 13B and 445N5, (gap between contig 4 and contig 13) was under $1 \mathrm{~kb}$ and was immediately filled by direct sequencing. Interestingly, the PCR product from primers $15 \mathrm{~B}$ and $445 \mathrm{~N} 3$ was quite large and contained both $16 \mathrm{~S}$ and $23 \mathrm{~S}$ rDNA and was verified and sequenced using the same rDNA based primer-walking strategy described above (Figure 7, lane 4/15). Resolution of the rearrangement uncovers an approximately $200 \mathrm{~kb}$ assembly error in the draft genome, and these types of large-scale rearrangements 
1. Gap
Identification

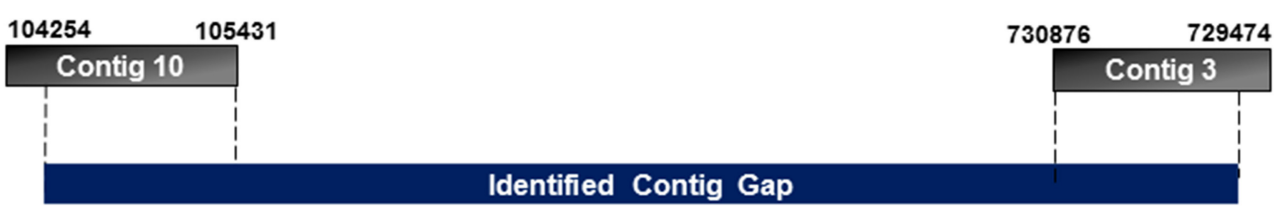

2. Primer
Design

3. Sequencingand Alignment

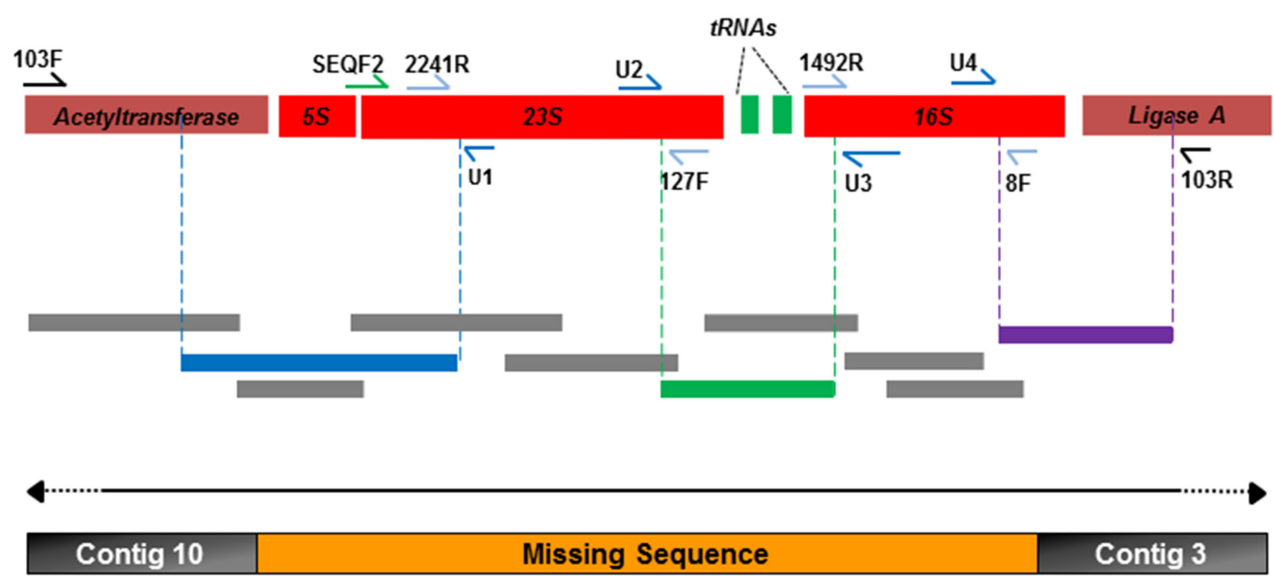

FIGURE 5 | Innovative primer walking strategy for sequencing of repetitive rRNA operons. Putative gaps between contigs were identified by the contig-reordering tool within Mauve against other completely sequenced P. polymyxa strains. Primers U1, U2, U3, and U4 were designed to amplify the rRNA operon as depicted, allowing for rapid resolution of the rRNA gene flanking sequences and intragenic regions. Primers depicted were used as sequencing primers for Sanger sequencing of unknown regions and the resulting sequences were overlapped to identify missing sequence. Sequences of the depicted primers are presented in Table $\mathbf{1}$

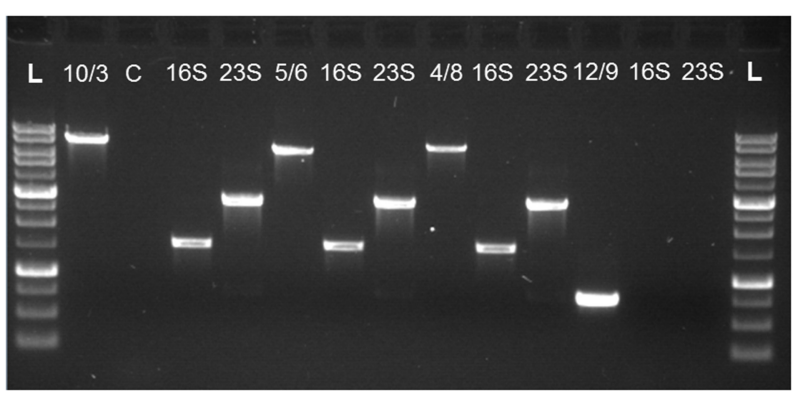

FIGURE 6 | Confirmation of the presence of rDNA operons located within contig gaps. PCR products joining the ends of two contigs were used as templates for PCR to determine presence of rRNA genes within the gaps. Gaps that tested positive for rRNA operons were closed using our innovative rRNA gap sequencing procedure as illustrated in Figure 4. Gaps that did not contain rRNA operons required traditional primer walking to determine the missing sequence. PCR products labeled as $16 \mathrm{~S}$ and $23 \mathrm{~S}$ rRNA genes were amplified from the respective preceding PCR product corresponding to the following gaps; $10 / 3$ - gap between contig 10 and contig 3,5/6 - gap between contig 5 and contig 6, 8/4 - gap between contig 8 and contig 4, 12/9 - gap between contig 12 and contig 9 . L represents a $1 \mathrm{~kb}$ molecular weight ladder and $\mathrm{C}$ is a representative template control utilizing distant primers not expected to be contained within the 10/3 gap to confirm template purity.

are commonly present in published draft genomes (Latreille et al., 2007).

As a final validation of genome assembly, the sequence of the 38 contigs from the original draft assembly, including the contigs containing $16 \mathrm{~S}$ and $23 \mathrm{~S}$ rRNA gene fragments that were merged earlier, were aligned against the final contiguous assembly of the CR1 genome, which confirmed coverage of the entire draft in the final sequence.

\section{GENOME ANNOTATION}

The data contained in gene and protein annotations is ultimately the primary reasoning for genome sequencing. Automated in silico annotation suites rely on open reading frame predictions and homology searches against databases of characterized proteins. A multitude of automated annotation pipelines exist, each with their own respective algorithms, strengths, and limitations, and careful consideration must be taken when deciding which annotation suite to be used (Richardson and Watson, 2013). To provide consistency with other publically available genomes we utilized the NCBI Prokaryotic Genome Automatic Annotation Pipeline (PGAAP) for the annotation of the complete CR1 genome. The PGAAP uses a combination of Hidden Markov Model based gene prediction and a sequence similarity search against Entrez Protein Clusters, the Conserved Domain Database, and the Clusters of Orthologous Groups database. Annotations are automatically cross-referenced by homology to proteins contained in the NCBI database and are assigned standard locus tags, protein/gene names, and protein domain annotations. Annotation of the $P$. polymyxa strain CR1 genome using the PGAAP revealed 5283 protein-coding sequences, 36 rRNAs, 87 tRNAs and 103 pseudogenes. Despite the wealth of knowledge on structure-function relationships in prokaryotes, over $20 \%$ of the genes in the CR1 genome correspond to a COG category of 


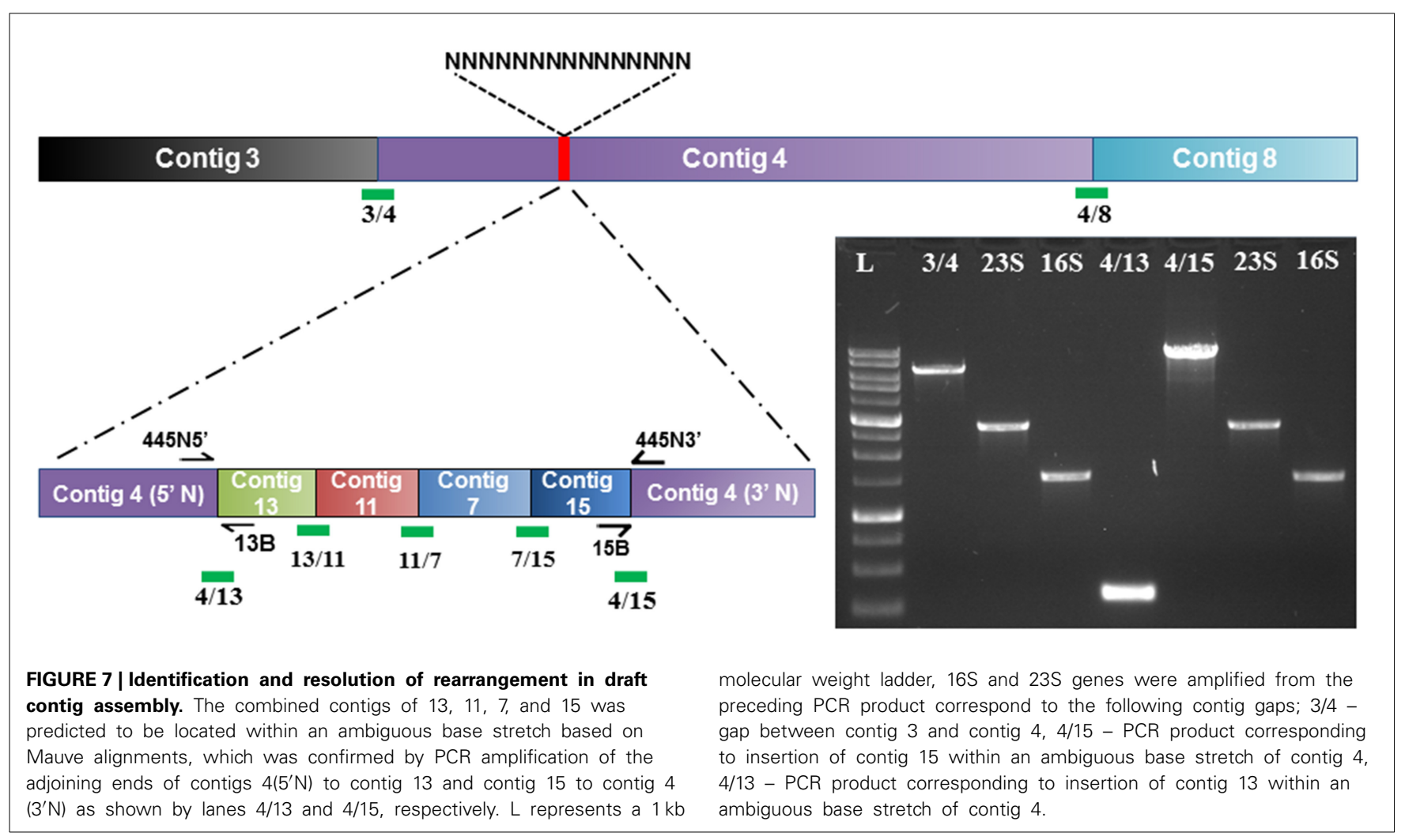

Table 2 | Specific Primers targeting identified contig gaps for amplification.

\begin{tabular}{lll}
\hline Contig gap & Forward primer & Reverse primer \\
\hline $5-1$ & AATAGACGGGTCTTCCTGCTTATAC & CGATACAACAGCCGACATTTGATTA \\
$5-6$ & ACCCTAATCGACTGCTTAATCTTGT & CATCTATTGAACTCATTGAACGGGG \\
$6-10$ & GATGAACCAAAACCTCACCAAGAAT & CGATTGGATCAAGATATTCGCTACG \\
$10-3$ & GCATCCACAATACGACCCATAAAAT & GAATGTAGGACGAACGGGTAAAATC \\
$3-4$ & CGGATTACCTCAAAAGGATTGGATG & AAAGAACCATCATGCACAGACAAAT \\
$12-1$ & GTGCCGTTCTAATGTGTAGCTTATC & AGATGGATAGTAGTCAGGCAAATCC \\
$9-2$ & GTATATCGGGGAAAGATAGGCAAT & GGTGTTTGTTGTAAGCTGTATGA \\
$8-4$ & TTGTTTGATACGTTTGGTACCTTGG & TCTCCAAGTCAGCGTTCGTC \\
$13-11$ & GACATGGTTGATTGAAAGTGACTGG & AGTGCTCAGCATGGAAGCAA \\
$11-7$ & TGGTCAATGTAAAACGCAATCTTCA & CCATCATATCCGGGCACCAA \\
$4-15$ & GCACTGAATAATCCCATTCTCAACC & TGAGGCAACAAGAATCCGCT \\
$4-15$ & ACATGTTGCTTTCTTTTGCTGA & GGACAACCAGGATACCGCAA \\
\end{tabular}

Each primer set was designed using Primer3 and tested for unintended priming using the Paenibacillus polymyxa strain CR1 draft genome as reference. Putative

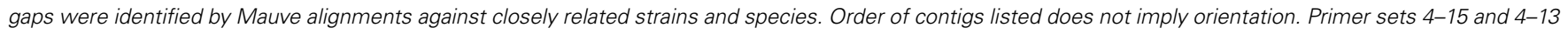
were designed to confirm the identified rearrangement as described in Figure 7.

general function prediction or unknown function (Eastman et al., 2014b).

\section{DISCUSSION}

Regardless of the breakthroughs in sequencing technologies in the preceding decade and the power of second-generation sequencing platforms, generation of a complete, finished bacterial genome remains a difficult task. Draft genome contiguity is limited by the presence of repetitive genetic elements longer than shortinsert and mate-pair read lengths, such as multiple copy and highly similar rDNA operons and tRNAs. Many sequencing and assembly errors inherent to second-generation sequencing technologies are present in draft assemblies as evidenced by a large number of ambiguous base stretches (Figure 4) and rearrangements (Figure 7) in the CR1 draft genome. The identification of rRNA gene fragments in a large proportion of contig ends 


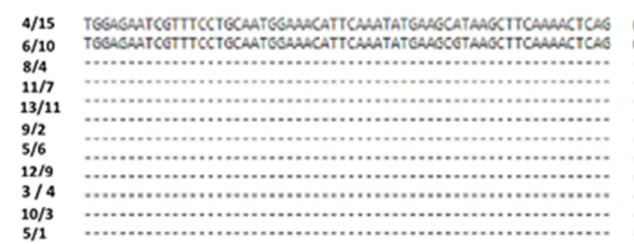

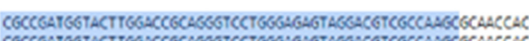

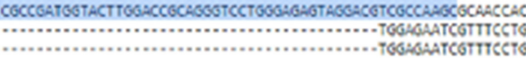
G.

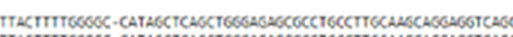

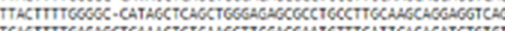

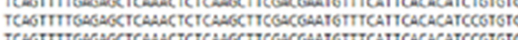
TCAOTITTGSAGCTCAACTCTCHOCTTCOACGATOTTTCATTCACACATCCGTGTS

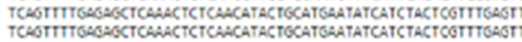

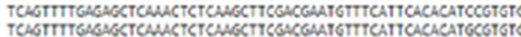
TCAGITTTGQAGCTCAACTCTCAGCTTCGACGATGITTCATTCACACATCCGTOTO

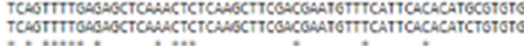

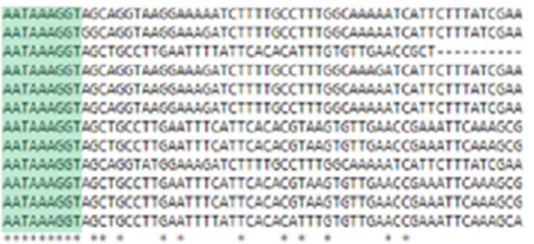

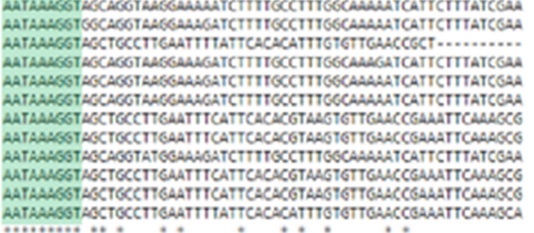

FIGURE 8 |Clustal $\Omega$ alignment of the $16 \mathrm{~S}$ and $23 \mathrm{~S}$ ribosomal subunit intragenic region of the CR1 genome. Numbers to the left of sequence data represent the specific contig gap where the ribosomal subunit DNA was located. CR1 encoded ribosomal subunit DNA was unanimously localized to gaps from the draft genome. Intragenic DNA sequences were obtained using primers $\mathrm{U} 2$ and $\mathrm{U} 3$ and sequenced by Sanger sequencing and aligned by Clustal $\Omega$ using default parameters. Sequence highlighted in blue corresponds to $5 S$ ribosomal subunit DNA, highlighted in green represents a highly conserved $P$. polymyxa species-specific 89 nt sequence.
181

56-23S
$117-235$
$103-23 S$
$610-23 S$
$84-23 S$
$1311-23 S$
$121-23 S$
$34-23 S$
$51-235$

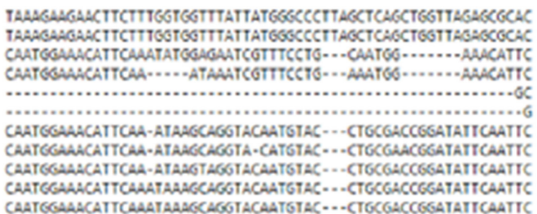

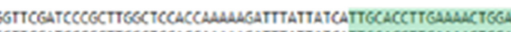

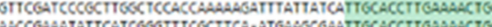

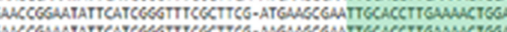

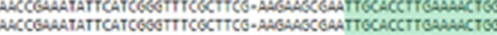

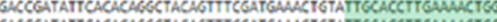

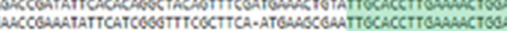

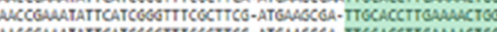

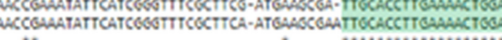

CATCGACATIITCITCTITGaAaseasagICT

catcGacatritici

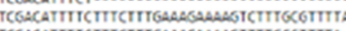

MTCGTATTTACCATGTAaAtaCT.

CATCGACATIITC

A tcgtatrtaccatgraat.....

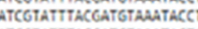

CCCTGATAAGGGTGAGGTCGGTGGTT GQGTCCACTAGGGCCCACCATATACTITATAT

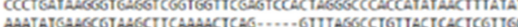

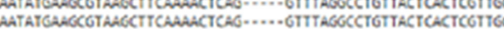

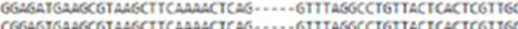

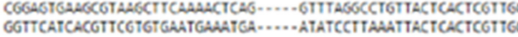

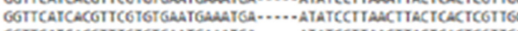

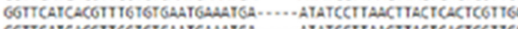

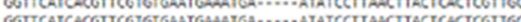

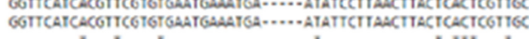

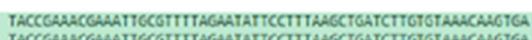

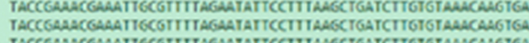

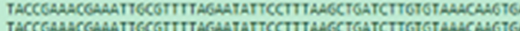

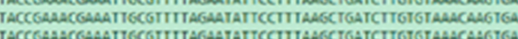

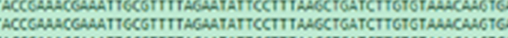

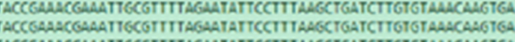

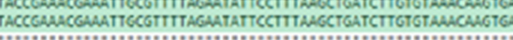
300701

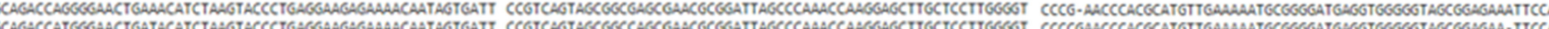

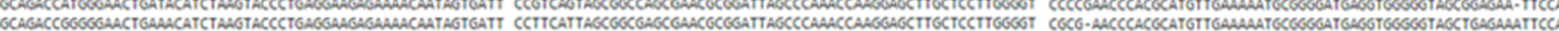

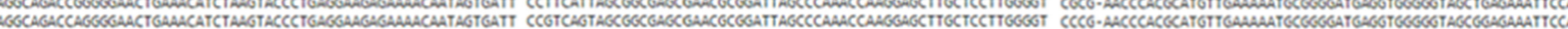

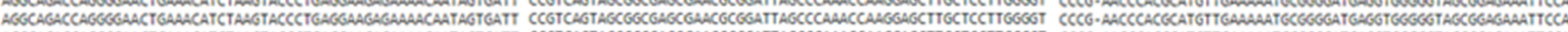

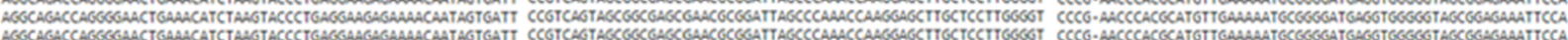

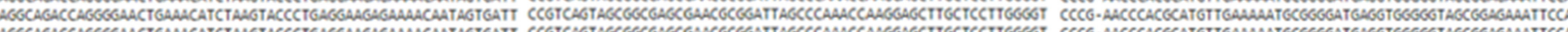

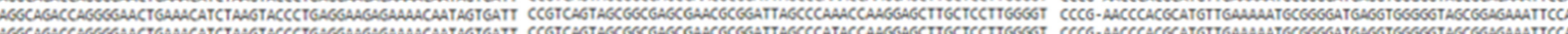
1061

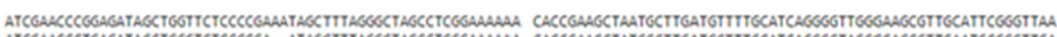

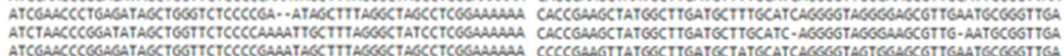

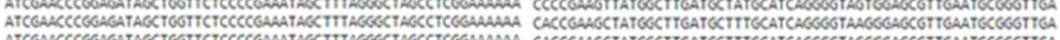

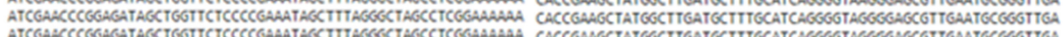

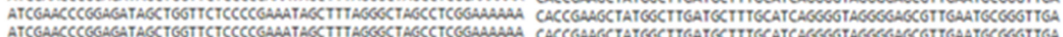

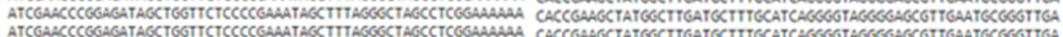

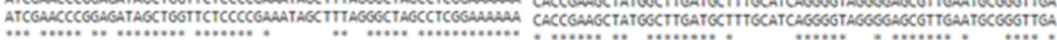
1241

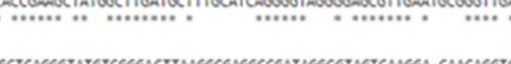

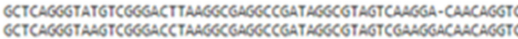

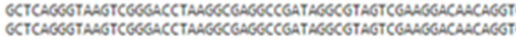

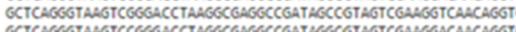

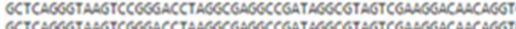

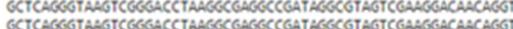

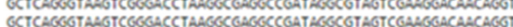

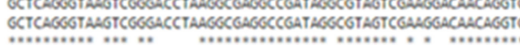

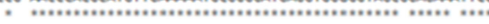

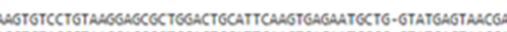

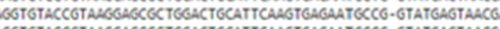

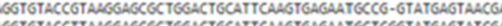

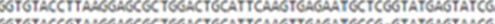

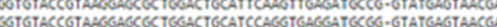

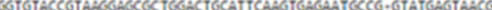

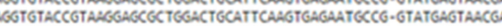

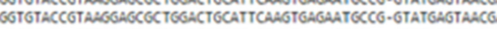
AAGGATTGTGAGATCAGATCCGCCGAHGGCTTALGGTTCC.- TGAGGAGGATCG TCC

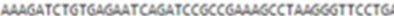

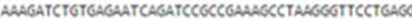

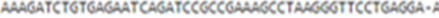

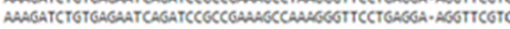

FIGURE 9 | Clustal $\Omega$ alignment of 235 ribosomal subunit regions from CR1 genome. Numbers to the left of sequence data represent the specific contig gap where the ribosomal subunit DNA was located. Numbers above the nucleotide sequence data represent the position of each $60 \mathrm{nt}$ frame. Regions not shown in the figure correspond to stretches of identical nucleotides between the depicted genes. 
in the draft CR1 genome reinforce previous findings that these types of repetitive genetic elements are a major limiting factor for in silico assembly (Rainey et al., 1996; Ricker et al., 2012). In addition, ribosomal subunit intragenic sequences are variable within an individual genome and it cannot be assumed that all rDNA operons have an identical composition (Figures 8, 9). Thus, the variety of features that need to be addressed during genome finishing requires a large amount of labor and resources to intensely scrutinize the draft genome sequence and experimentally confirm the correct assembly, arrangement, and structure.

The continued development and availability of simple and effective genome finishing strategies is paramount to accommodate the persistent onslaught of advances in sequencing technologies. Without concomitant advances in genome finishing strategies we cannot fully explore the nuances of evolution, phylogenetics, and prokaryotic genomic structure that can only be obtained from completed genomes.

As the costs of de novo genome sequencing continue to decrease, de novo genome sequencing will become increasingly common in the characterizations of bacterial strains. Based on the repetitive and conserved nature of rDNA operons we designed a procedure that allows for precise identification and sequencing of rDNA sequences within contig gaps. Using our procedure multiple primer walking sequencing reactions can be performed in parallel to target repetitive rDNA operons, thereby effectively reducing the time and resources needed for gap closure and assembly of scaffolded bacterial genomes. Despite recent developments in long read and single molecule sequencing technologies, due to the low base calling accuracy and high costs it is unlikely these technologies will completely replace Illumina sequencing for bacterial genome sequencing in the near future (Quail et al., 2012). Our approach increases the feasibility and speed of genome sequencing and offers an economical and technically simple strategy for microbial genome finishing, especially when available manpower and resources is a limiting factor in the decision to finish a genome.

\section{NUCLEOTIDE SEOUENCE ACCESSION NUMBERS}

The complete genomic sequences of strains referenced in the text are publically available in the NCBI GenBank database with the following accession numbers; P. polymyxa strain CR1 NC_023037.1, P. polymyxa strain E681 - NC_014483.1, P. terrae strain HPL-003 - NC_016641.1.

\section{AUTHOR CONTRIBUTIONS}

Alexander W. Eastman and Ze-Chun Yuan conceived and designed the study and drafted the manuscript. Alexander W. Eastman performed the data collection and analysis. Both authors approved the final manuscript.

\section{ACKNOWLEDGMENTS}

We would like to thank Naeem Nathoo and Brian Weselowski for editing of the manuscript and technical support. This research was funded by the Agriculture and Agri-Food Canada, Growing Forward-AgriFlex funding (RBPI number 2555), and conducted by the authors as part of their duties. We really appreciate
Dr. Frank T. Robb, University of Maryland, USA, for editing of our manuscript. We also appreciate Dr. Jonathan $\mathrm{H}$. Badger from J. Craig Venter Institute, USA, and Dr. Viktoria Shcherbakova from Russian Academy of Sciences, Russia for reviewing our manuscript and their valuable comments to improve the manuscript.

\section{REFERENCES}

Anzai, Y., Kim, H., Park, J.-Y., Wakabayashi, H., and Oyaizu, H. (2000). Phylogenetic affiliation of the Pseudomonads based on 16S rRNA sequence. Int. J. Syst. Evol. Microbiol. 50, 1563-1589. doi: 10.1099/00207713-50-4-1563

Bentley, S. (2010). Taming the next-gen beast. Nat. Rev. Microbiol. 8, 161. doi: $10.1038 /$ nrmicro2322

Birol, I., Jackman, S. D., Nielsen, C. B., Qian, J. Q., Varhol, R., Stazyk, G., et al. (2009). De novo transcriptome assembly with ABySS. Bioinformatics 25, 2872-2877. doi: 10.1093/bioinformatics/btp367

Carver, T., Thomson, N., Bleasby, A., Berriman, M., and Parkhill, J. (2009). DNAPlotter: circular and linear interactive genome visualization. Bioinformatics 25, 119-120. doi: 10.1093/bioinformatics/btn578

Darling, A. E., Mau, B., and Perna, N. T. (2010). progressiveMauve: multiple genome alignment with gene gain, loss and rearrangement. PloS ONE 5:e11147. doi: 10.1371/journal.pone.0011147

Darling, A. E., Tritt, A., Eisen, J. A., and Facciotti, M. T. (2011). Mauve assembly metrics. Bioinformatics 27, 2756-2757. doi: 10.1093/bioinformatics/btr451

Delseny, M., Han, B., and Ie, Y. (2010). Plant science high throughput DNA sequencing: the new sequencing revolution. Plant Sci. 179, 407-422. doi: 10.1016/j.plantsci.2010.07.019

Eastman, A. W., Heinrichs, D. E., and Yuan, Z.-C. (2014b). Comparative and genetic analysis of the four sequenced Paenibacillus polymyxa genomes reveals a diverse metabolism and conservation of genes relevant to plant-growth promotion and competitiveness. BMC Genomics 15:851. doi: 10.1186/14712164-15-851

Eastman, A. W., Weselowski, B., Nathoo, N., and Yuan, Z.-C. (2014a). Complete genome sequence of Paenibacillus polymyxa CR1, a plant growth-promoting bacterium isolated from the corn rhizosphere exhibiting potential for biocontrol, biomass degradation, and biofuel. Genome Announ. 2, e01218-13. doi: 10.1128/genomeA.01218-13

Ewing, B., and Green, P. (1998). Base-calling of automated sequencer traces using Phred. II. Error probabilities. Genome Res. 8, 186-194. doi: 10.1101/gr. 8.3.175

Ewing, B., Hillier, L., Wendl, M. C., and Green, P. (1998). Base-calling of automated sequencer traces using Phred. I. Accuracy assessment. Genome Res. 8, 175-185. doi: $10.1101 /$ gr.8.3.175

Gibbons, J. G., Janson, E. M., Hittinger, C. T., Johnston, M., Abbot, P., and Rokas, A. (2009). Benchmarking next-generation transcriptome sequencing for functional and evolutionary genomics. Mol. Biol. Evol. 26, 2731-2744. doi: $10.1093 / \mathrm{molbev} / \mathrm{msp} 188$

Glenn, T. C. (2011). Field guide to next-generation DNA sequencers. Mol. Ecol. Resour. 11, 759-769. doi: 10.1111/j.1755-0998.2011.03024.x

Gritsenko, A. A., Nijkamp, F. J., Reinders, M. J. T., and de Ridder, D. (2012). GRASS: a generic algorithm for scaffolding next-generation sequencing assemblies. Bioinformatics 28, 1429-1437. doi: 10.1093/bioinformatics/bts 175

Horner, D. S., Pavesi, G., Castrignano, T., Onorio De Meo, P. D., Liuni, S., Sammeth, M., et al. (2009). Bioinformatics approaches for genomics and post genomics applications of next-generation sequencing. Brief. Bioinformatics 11, 181-197. doi: 10.1093/bib/bbp046

Hunt, D. E., Klepac-Ceraj, V., Acinas, S. G., Gautier, C., Bertilsson, S., and Polz, M. F. (2006). Evaluation of $23 \mathrm{~S}$ rRNA PCR primers for use in phylogenetic studies of bacterial diversity. Appl. Environ. Microbiol. 72, 2221-2225. doi: 10.1128/AEM.72.3.2221-2225.2006

Hurt, R. A., Brown, S. D., Podar, M., Palumbo, A. V., and Elias, D. A. (2012). Sequencing intractable DNA to close microbial genomes. PloS ONE 7:e41295. doi: 10.1371/journal.pone.0041295

Kim, J. F., Jeong, H., Park, S.-Y., Kim, S.-B., Park, Y. K., Choi, S.-K., et al. (2010). Genome sequence of the polymyxin-producing plant-probiotic rhizobacterium Paenibacillus polymyxa E681. J. Bacteriol. 192, 6103-6104. doi: 10.1128/JB.00983-10 
Lal, S., and Tabacchioni, S. (2009). Ecology and biotechnological potential of Paenibacillus polymyxa: a minireview. Indian J. Microbiol. 42, 2-10. doi: 10.1007/s12088-009-0008-y

Latreille, P., Norton, S., Goldman, B. S., Henkhaus, J., Miller, N., Barbazuk, B., et al. (2007). Optical mapping as a routine tool for bacterial genome sequence finishing. BMC Genomics 8:321. doi: 10.1186/1471-2164-8-321

Li, S., Yang, D., Qiu, M., Shao, J., Guo, R., Shen, B., et al. (2014). Complete genome sequence of Paenibacillus polymyxa SQR-21, a plant growth-promoting rhizobacterium with antifungal activity and rhizosphere colonization ability. Genome Announ. 2, e00281-14. doi: 10.1128/genomeA.00281-14

Lin, S.-H., and Liao, Y.-C. (2013). CISA: contig integrator for sequence assembly of bacterial genomes. PloS ONE 8:e60843. doi: 10.1371/journal.pone. 0060843

Luo, R., Liu, B., Xie, Y., Li, Z., Huang, W., Yuan, J., et al. (2012). SOAPdenovo2: an empirically improved memory-efficient short-read de novo assembler. GigaScience 1, 1-6. doi: 10.1186/2047-217X-1-18

Ma, M., Wang, C., Ding, Y., Li, L., Shen, D., Jiang, X., et al. (2011). Complete genome sequence of Paenibacillus polymyxa SC2, a strain of plant growth-promoting rhizobacterium with broad-spectrum antimicrobial activity. J. Bacteriol. 193, 311-312. doi: 10.1128/JB.01234-10

Maclean, D., Jones, J. D. G., and Studholme, D. J. (2009). Application of 'nextgeneration' sequencing technologies to microbial genetics. Nat. Rev. Microbiol. 7, 287-296. doi: 10.1038/nrmicro2088

Mardis, E., McPherson, J., Martienssen, R., Wilson, R. K., and McCombie, R. W. (2002). What is finished, and why does it matter? Genome Biol. 12, 669-671. doi: 10.1101 /gr.032102

McGinn, S., and Gut, I. G. (2013). DNA sequencing - spanning the generations. New Biotechnol. 30, 366-372. doi: 10.1016/j.nbt.2012.11.012

Medini, D., Serruto, D., Parkhill, J., Relman, D. A., Donati, C., Moxon, R., et al. (2008). Microbiology in the post-genomic era. Nat. Rev. Microbiol. 6, 419-430. doi: 10.1038/nrmicro1901

Miller, J. R., Delcher, A. L., Koren, S.,Venter, E., Walenz, B. P., Brownley, A., et al. (2008). Aggressive assembly of pyrosequencing reads with mates. Bioinformatics 24, 2818-2824. doi: 10.1093/bioinformatics/btn548

Morey, M., Fernández-Marmiesse, A., Castiñeiras, D., Fraga, J. M., Couce, M. L., and Cocho, J. A. (2013). A glimpse into past, present, and future DNA sequencing. Mol. Genet. Metab. 110, 3-24. doi: 10.1016/j.ymgme.2013. 04.024

Nagarajan, N., Cook, C., Di Bonaventura, M., Ge, H., Richards, A., Bishop-Lilly, K. A., et al. (2010). Finishing genomes with limited resources: lessons from an ensemble of microbial genomes. BMC Genomics 11:242. doi: 10.1186/14712164-11-242

Niu, B., Rueckert, C., Blom, J., Wang, Q., and Borriss, R. (2011). The genome of the plant growth-promoting rhizobacterium Paenibacillus polymyxa M-1 contains nine sites dedicated to nonribosomal synthesis of lipopeptides and polyketides. J. Bacteriol. 193, 5862-5863. doi: 10.1128/JB.05806-11

Pop, M., Kosack, D. S., and Salzberg, S. L. (2004). Hierarchical scaffolding with bambus. Genome Res. 14, 149-159. doi: 10.1101/gr.1536204

Poptsova, M. S., and Gogarten, J. P. (2010). Using comparative genome analysis to identify problems in annotated microbial genomes. Microbiology 156 1909-1917. doi: 10.1099/mic.0.033811-0

Quail, M. A., Smith, M., Coupland, P., Otto, T. D., Harris, S. R., Connor, S. R., et al. (2012). A tale of three next generation sequencing platforms: comparison of
Ion Torrent, Pacific Biosciences and Illumina MiSeq sequencers. BMC Genomics 13:341. doi: 10.1186/1471-2164-13-341

Rainey, F. A., Ward-Rainey, N. L., Janssen, P. H., Hippe, H., and Stackebrandt, E. (1996). Clostridium paradoxum DSM730AT contains multiple 16s rRNA genes with heterogeneous intervening sequences. Microbiology 142, 2087-2095. doi: 10.1099/13500872-142-8-2087

Richardson, E. J., and Watson, M. (2013). The automatic annotation of bacterial genomes. Brief. Bioinformatics 14, 1-12. doi: 10.1093/bib/bbs007

Ricker, N., Qian, H., and Fulthorpe, R. R. (2012). The limitations of draft assemblies for understanding prokaryotic adaptation and evolution. Genomics 100, 167-175. doi: 10.1016/j.ygeno.2012.06.009

Rutherford, K., Parkhill, J., Crook, J., Horsnell, T., and Rice, P. (2000). Artemis: sequence visualization and annotation. Bioinformatics 16, 944-945. doi: 10.1093/bioinformatics/16.10.944

Salzberg, S. L., and Yorke, J. A. (2005). Beware of mis-assembled genomes. Bioinformatics 21, 4320-4321. doi: 10.1093/bioinformatics/bti769

Schuster, S. C. (2008). Next-generation sequencing transforms today's biology. Nat Methods 5, 16-18. doi: 10.1038/nmeth1156

Shin, S. H., Kim, S., Kim, S. Y., Young, H., Cho, S. J., Kim, D. R., et al. (2012). Genome sequence of Paenibacillus terrae HPL-003, a xylanase-producing bacterium isolated from soil found in forest residue. J. Bacteriol. 194, 1266. doi: 10.1128/JB.06668-11

Tsai, I. J., Otto, T. D., and Berriman, M. (2010). Improving draft assemblies by iterative mapping and assembly of short reads to eliminate gaps. Genome Biol. 11, R41. doi: 10.1186/gb-2010-11-4-r41

Wetzel, J., Kingsford, C., and Pop, M. (2011). Assessing the benefits of using matepairs to resolve repeats in de novo short-read prokaryotic assemblies. BMC Bioinformatics 12:95. doi: 10.1186/1471-2105-12-95

Yu, B., Sun, J., Bommareddy, R. R., Song, L., and Zeng, A.-P. (2011). Novel (2R, 3R)-2, 3-butanediol dehydrogenase from potential industrial strain Paenibacillus polymyxa ATCC 12321. Appl. Environ. Microbiol. 77, 4230-4233. doi: 10.1128/AEM.02998-10

Zerbino, D. R., and Birney, E. (2008). Velvet: algorithms for de novo short read assembly using de Bruijn graphs. Genome Res. 18, 821-829. doi: 10.1101/gr.074492.107

Conflict of Interest Statement: The authors declare that the research was conducted in the absence of any commercial or financial relationships that could be construed as a potential conflict of interest.

Received: 07 November 2014; accepted: 16 December 2014; published online: 21 January 2015.

Citation: Eastman AW and Yuan Z-C (2015) Development and validation of an rDNA operon based primer walking strategy applicable to de novo bacterial genome finishing. Front. Microbiol. 5:769. doi: 10.3389/fmicb.2014.00769

This article was submitted to Evolutionary and Genomic Microbiology, a section of the journal Frontiers in Microbiology.

Copyright (C) 2015 Eastman and Yuan. This is an open-access article distributed under the terms of the Creative Commons Attribution License (CC BY). The use, distribution or reproduction in other forums is permitted, provided the original author(s) or licensor are credited and that the original publication in this journal is cited, in accordance with accepted academic practice. No use, distribution or reproduction is permitted which does not comply with these terms. 